\title{
Structure of CdTe/ZnTe superlattices
}

R. H. Miles and T. C. McGill

California Institute of Technology, Pasadena, California 91125

S. Sivananthan, X. Chu, and J. P. Faurie

Department of Physics, University of Illinois-Chicago Circle, Chicago, Illinois 60680

(Received 20 March 1987; accepted 15 April 1987)

The structure of $\mathrm{CdTe} / \mathrm{ZnTe}$ superlattices has been analyzed through $\theta / 2 \theta \mathrm{x}$-ray diffraction, photoluminescence, and in situ reflection high-energy electron diffraction (RHEED) measurements. Samples are found to break away from $\mathrm{Cd}_{x} \mathrm{Zn}_{1_{-x}} \mathrm{Te}$ buffer layers as a consequence of the $6 \%$ lattice mismatch in this system. However, defect densities in these superlattices are seen to drop dramatically away from the buffer layer interface, accounting for the intense photoluminescence and high-average strain fields seen in each of our samples. Observed variations in residual strains suggest that growth conditions play a role in forming misfit defects. This could explain discrepancies with calculated values of critical thickness based on models which neglect growth conditions. Photoluminescence spectra reveal that layer-to-layer growth proceeded with single monolayer uniformity, suggesting highly reproducible growth. Our results give hope for relatively defect-free $\mathrm{Cd}_{x} \mathrm{Zn}_{1-x} \mathrm{Te} / \mathrm{Cd}_{y} \mathrm{Zn}_{1-y} \mathrm{Te}$ superlattices with the potential for applications to optoelectronics offered by intense visible light emitters.

\section{INTRODUCTION}

Successful growth of type II-VI superlattices composed of highly lattice-mismatched layers has recently been demonstrated. ${ }^{-4}$ While these superlattices display potential for applications to optoelectronics by virtue of their large direct band gaps, they have drawn into question the degree to which strain can be accommodated within a multilayer structure. Devices based on these structures will display properties and performance determined to a large extent by the degree to which grown-in stresses can be accommodated by elastic strain. ${ }^{5}$ For this reason it has become important to examine the critical thicknesses to which these superlattices can be grown before misfit defects are formed.

We present structural studies of a number of $\mathrm{CdTe} / \mathrm{ZnTe}$ superlattices. CdTe and $\mathrm{ZnTe}$ possess bulk lattice constants differing by $6 \%$ from each other, creating enormous internal stresses characteristic of large-band-gap II-VI superlattices. In this paper we examine the accommodation of these stresses through elastic strain and misfit defects, the consequences of growth on different $\mathrm{Cd}_{x} \mathrm{Zn}_{1-x}$ Te buffer layers, and the reproducibility of layer-to-layer growth. Section II describes three independent experiments. We first present results derived from photoluminescence spectra. This is followed by $x-$ ray diffraction data accumulated for each of our samples. A brief discussion of the interpretation of $\mathrm{x}$-ray measurements for strained-layer superlattices is also included. The section closes with results of in situ reflection high-energy electron diffraction (RHEED) measurements made during growth of a single superlattice sample. Our results are compared with calculated critical thicknesses in Sec. III.

\section{EXPERIMENT}

The samples used in this study were grown by molecularbeam epitaxy (MBE) in a Riber 2300. Superlattice sample characteristics are listed in Table I. Individual CdTe and $\mathrm{ZnTe}$ layers were grown to thicknesses of 20-55 $\AA$. Each sample consisted of several hundred periods, which resulted in superlattices $1-2 \mu \mathrm{m}$ thick. Samples were grown on a variety of buffer layers to examine modulations of strain resulting from growth on different templates.

Photoluminescence experiments performed on these samples have been described elsewhere. ${ }^{3}$ In brief, samples were excited optically at energies greater than the sample band gaps. Spectra were accumulated at temperatures down to $2 \mathrm{~K}$ in a helium immersion dewar. Typical luminescence spectra from a CdTe/ZnTe superlattice and $\mathrm{Cd}_{x} \mathrm{Zn}_{1-x} \mathrm{Te}$ alloy are shown in Fig. 1. Each superlattice displays intense, visible luminescence orders of magnitude brighter than that from corresponding $\mathrm{Cd}_{x} \mathrm{Zn}_{1-x} \mathrm{Te}$ alloys. This enhancement in luminescent efficiency is often observed in highquality superlattices ${ }^{6-8}$ and has been attributed variously to carrier confinement in quantum wells, ${ }^{\circ}$ defect and impurity gettering at interfaces, ${ }^{7}$ and recuctions in surface recombination velocities. ${ }^{8}$ Our spectra are dominated by a single intense peak which has been attributed to an exciton. ${ }^{9}$ Peaks display typical linewidths of about $30 \mathrm{meV}$. This energy corresponds to the change in superlattice-band gap due to a single monolayer tiluctuation in CdTe-well width, which suggests that layer-to-layer growth proceeded in a highly

TABLE I. CdTe-ZnTe superlattice sample characteristics.

\begin{tabular}{lccc}
\hline Sample & $\begin{array}{c}\text { Layer thicknesses } \\
\text { (CdTe/ZnTe) }(\AA)\end{array}$ & Periods & Top buffer layer \\
\hline 1 & $26 / 32$ & 200 & $\mathrm{CdTe}$ \\
2 & $31 / 23$ & 200 & $\mathrm{CdTe}$ \\
3 & $56 / 50$ & 150 & $\mathrm{CdTe}$ \\
4 & $27 / 30$ & 200 & $\mathrm{ZnTe}$ \\
5 & $24 / 30$ & 200 & $\mathrm{Cd}_{0.46} \mathrm{Zn}_{0.54} \mathrm{Te}$ \\
6 & $27 / 30$ & 200 & $\mathrm{Cd}_{0.41} \mathrm{Zn}_{0.59} \mathrm{Te}$ \\
7 & $35 / 32$ & 200 & $\mathrm{Cd}_{0.46} \mathrm{Zn}_{0.54} \mathrm{Te}$ \\
8 & $21 / 20$ & 400 & $\mathrm{Cd}_{0.50} \mathrm{Zn}_{0.50} \mathrm{Te}$ \\
\hline
\end{tabular}




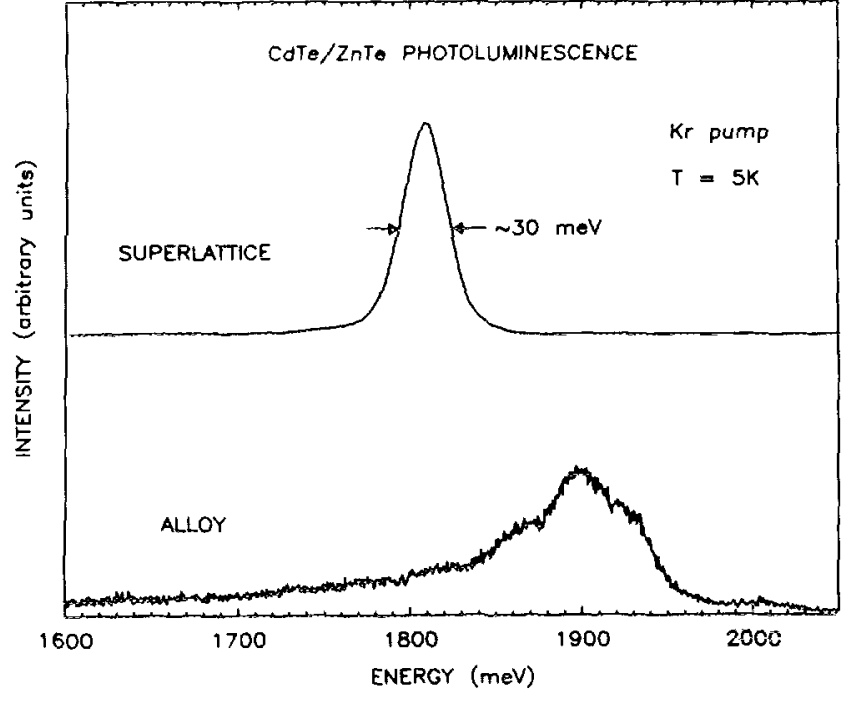

Fig. 1. Photoluminescence spectra from superlattice sample 2 and a $\mathrm{Cd}_{\mathrm{O} n} \mathrm{Zn}_{\mathrm{n}, \mathrm{n}} \mathrm{Te}$ alloy at $5 \mathrm{~K}$. Spectra are plotted on different verticai scales.

reproducible manner. In addition, the absence of fiuctuations as the incident beam was scanned across the surface of the sample suggests a high degree of uniformity in the plane of growth.

Photoluminescence data have yielded approximate values for the band gap of each sample studied. Comparing these experimental values with theory has yielded rough limits on the strain distributions present in our samples. ${ }^{3}$ Strain is known to play a role in determining the band structure of crystals. ${ }^{10}$ In the $\mathrm{CdTe} / \mathrm{ZnTe}$ system strain splits the lightand heavy-hole valence bands and causes a shift in band gap of the material. This effect can be quite pronounced; different distributions of strain should produce band-gap shifts of up to $350 \mathrm{meV}$ in the samples studied. The absence of these shifts has demonstrated that those of our samples grown on pure CdTe do not have strain distributions derived from the CdTe-buffer layer.

Luminescence intensities are high for all samples, regardless of the buffer layers used. Although it is difficult to make quantitative connections between defect densities and luminescent intensities, high-defect densities typicalily open strong nonradiative decay channels. ${ }^{5}$ This suggesis that the large number of misfit defects introduced at the buffer layer interfaces do not travel far into the superlattices. Our optical penetration depths were short $(<1 \mu \mathrm{m})$, suggesting that growth was of high quality about $1 \mu \mathrm{m}$ away from the superlattice/buffer-layer interface. This conjecture is supported by transmission-electron microscope (TEM) measurements 11 on sample 8 , which show $60^{\circ}$ dislocation densities dropping from $10^{10}-10^{11} \mathrm{~cm}^{-2}$ near the interface to about $10^{8} \mathrm{~cm}^{-2}$ near the top surface of the superlattice.

Strain distributions have been further analyzed through $\mathrm{x}$ ray diffraction spectra accumulated for each of the samples. Diffraction of $\mathrm{Cu} K \alpha$ x-rays was measured in a $\theta / 2 \theta$ arrangement. Since the geometry used was symmetric about each sample's growth axis, we were probing lattice constants only in the growth direction. As depicted in Fig. 2, growth of two lattice-mismatched materials at a single in-plane lattice
COHERENTLY STRAINED GROWTH

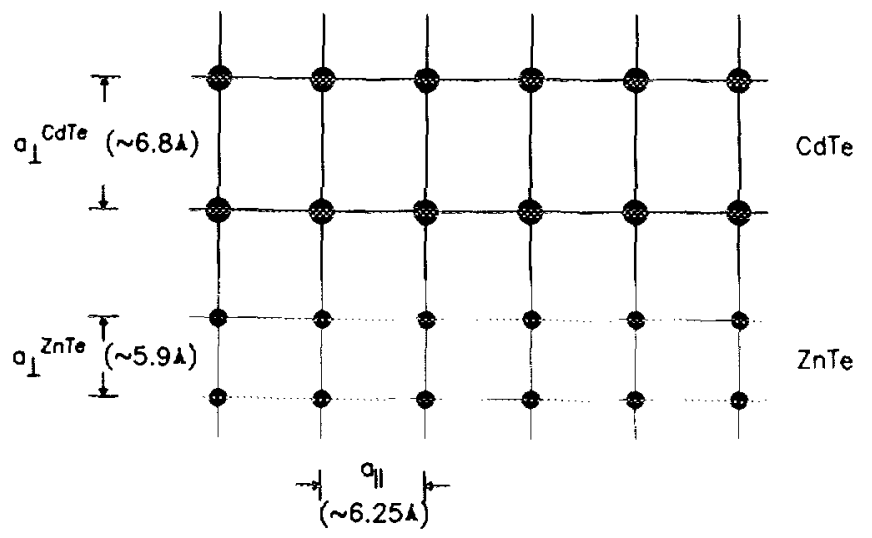

Fig. 2. Exaggerated view of unit cell deformation at a coherently strained $\mathrm{CdTe} / \mathrm{ZnTe}$ interface. Growth at a single in-plane lattice constant results in a reduction of the growth-direction lattice parameter in $\mathrm{ZnTe}$ and a uniaxial dilation in $\mathrm{CdTe}$.

constant results in tetragonal distortion. Measurement of the growth-direction lattice parameters reveals the degree of distortion in the structure and hence the amount of strain present.

Spectra revealed (200)-like and (400)-like superlattice peaks (referred to the cubic CdTe or $\mathrm{ZnTe}$ unit cells), as well as single peaks attributable to $\mathrm{Cd}_{x} \mathrm{Zn}_{1-x}$ Te buffer layers and to GaAs substrates. Experimental results have been compared with calculated spectra derived in the kinematical approximation. This is a good approximation when $x$-ray penetration depths are long compared to the size of the crystal being analyzed, as is the case in our experiment.

In the kinematical approximation, the structure of an $x$ ray diffraction spectrum is calculated by considering the contributions from a single unit cell and then summing these contributions across the extent of the crystal. For the case of diffraction from a superlattice, in which each unit cell is composed of several layers of one material followed by several layers of another, the intensity of diffracted $x$ rays, $I$, can be written as

$$
\begin{gathered}
I \propto \mid\left(\sum_{n}^{N} e^{i k n A_{2}}\right)\left[\left(\sum_{m_{1}}^{M_{1}} e^{i k m_{1} a_{1}}\right)\left(\sum_{j_{1}}^{J_{1}} f_{j_{1}} e^{i k r_{z}^{\prime}}\right)\right. \\
+ \text { similar terms for material } 2]\left.\right|^{2} \\
\propto \frac{\sin ^{2}\left(N k A_{2}\right)}{\sin ^{2}\left(k A_{z}\right)} \mid \frac{\sin \left(M_{1} k a_{1}\right)}{\sin \left(k a_{1}\right)} \sum_{j_{1}}^{J_{1}} f_{j_{1}} e^{i k r_{z}^{\prime}} \\
\quad+\text { similar terms for material }\left.2\right|^{2} .
\end{gathered}
$$

In these equations, $f_{j}$ are the atomic factors, $r_{z}^{j}$ are the growth-direction coordinates of the atoms in a single unit cell, $a_{i}$ are the constituent material lattice constants, and $A_{z}$ is the length of the superlattice unit cell in the growth direction. The first sum extends over the number $N$ of superlattice unit cells, the second describes a single superlattice cell as a sum over $M$ constituent material unit cells, and the third describes atomic contributions to a standard bulk-like unit 
cell. From this construction it is apparent that the structure will be dominated by sharp, closely spaced peaks described by $\sin ^{2} N k A_{z} / \sin ^{2} k A_{z}$ which result from the requirement that scattered waves maintain coherence between superlattice unit cells. For small-period superlattices the diffraction condition arising from a single superlattice unit cell is not a stringent one. In particular, this condition appears as a slow modulation in amplitude of these narrow peaks. It should be noted that in a superlattice this slow modulation provides the only information relating to the lattice constants within each layer. The size of the superlattice unit cell is typically unrelated to the lattice constants of the constituent materials, hence the positions of the narrow peaks defined by this periodicity carry no information pertaining to these parameters.

Growth-direction lattice constants in the two materials forming the superlattice can only be deduced if the single unit cell diffraction envelopes associated with each material are separated sufficiently to be distinguished from each other. The widths of these envelopes can be approximated by Scherrer's formula, ${ }^{12}$

$$
\theta_{\mathrm{FWHM}} \simeq 0.9 \frac{\lambda}{L \cos \theta},
$$

where the thickness of each layer of material within a superlattice period is given by $L_{i}=M_{i} a_{i}$. Since positions of the two envelopes can be determined by Bragg's law we find that constituent lattice constants can only be separated if

$$
n\left|\frac{1}{a_{1}}-\frac{1}{a_{2}}\right|>0.9\left(\frac{1}{L_{1}}+\frac{1}{L_{2}}\right) \text {. }
$$

This condition is satisfied in our samples only when the growth-direction lattice constants deviate substantially from bulk values. Such deviations are to be expected in heavily strained lattices.

Diffraction spectra from two of the samples are shown in Figs. 3 and 4 . Also shown are calculated spectra for the cases of unstrained individual layers and for coherently strained

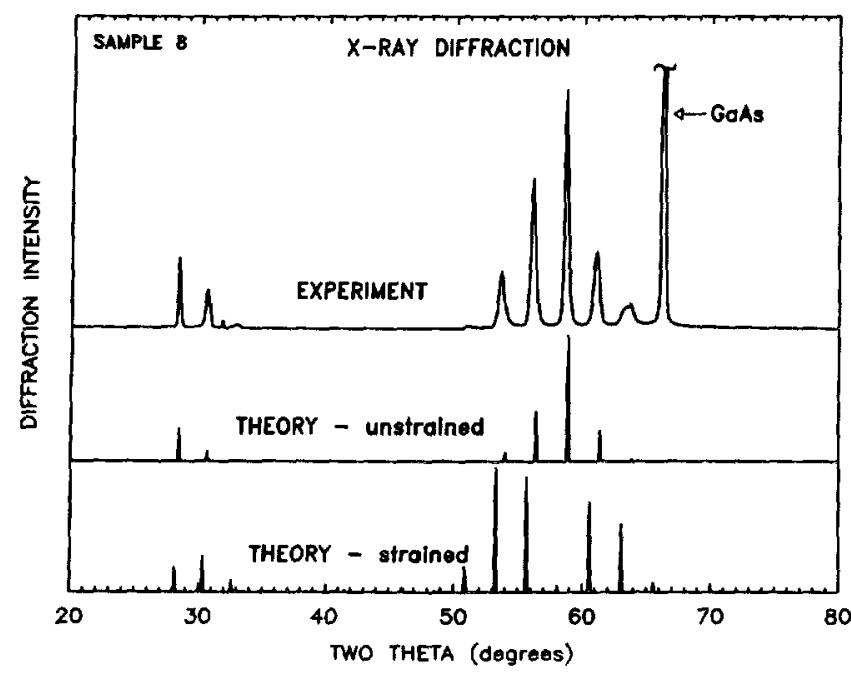

FIG. 3. $\theta / 2 \theta$-ray diffraction scan from superlattice sample 8 showing (200)-like and (400)-like diffraction peaks. The sample was irradiated with $\mathrm{Cu} K \alpha \mathrm{x}$-rays. Also shown are spectra calculated in the kinematical approximation for the cases of unstrained growth and free-standing strained growth.

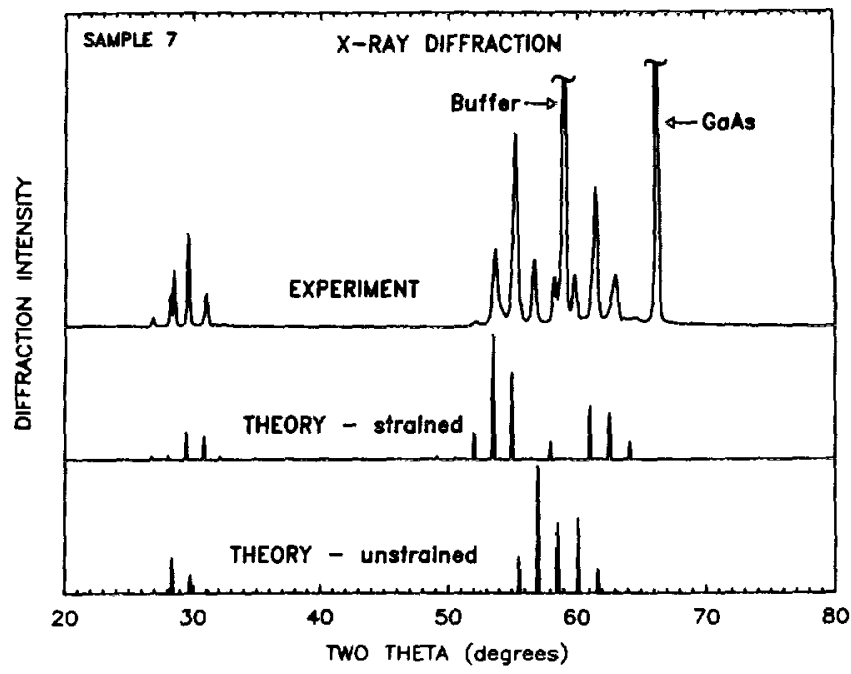

FIG. 4. $\theta / 2 \theta \mathrm{x}$-ray diffraction scan from superlattice sample 7. Note that (400)-like diffraction peaks around $2 \theta=58^{\circ}$ are divided into two envelopes. The sample was irradiated with $\mathrm{Cu} K \alpha \mathrm{x}$-rays. Also shown are spectra calculated in the kinematical approximation for the cases of unstrained growth and free-standing strained growth.

structures. Form factors used in these calculations were taken from Ref. 13. The spectra for coherently strained structures were calculated assuming in-plane lattice parameters intermediate to those of $\mathrm{CdTe}$ and $\mathrm{ZnTe}$ and appropriate to a free-standing (minimum energy) superlattice. The data shown in Fig. 3 appear to be in excellent agreement with theory when the structure is assumed to be unstrained. The most intense ( 400 )-like superlattice peak is enhanced by the superposition of diffraction from the buffer layer at this angle, but agreement with the unstrained limit is good nevertheless. Figure 4 shows diffraction representative of the remaining samples. These samples show high levels of residual strain, as evidenced by the appearance of two (400)-like envelopes, but are not in perfect agreement with calculated spectra appropriate to a free-standing structure. The envelopes are not separated as much as would be expected for a coherently strained structure; stress is clearly being accommodated by a combination of misfit defects and elastic strain. No attempt has been made to perform a quantitative fit to these experimental spectra by taking the growth direction lattice parameters as free variables. Such fits are of limited value once the existence of high-defect densities has been inferred since these spectra are fairly insensitive to inhomogeneous strain fields. X-ray penetration depths are on the order of microns, resulting in spectra that average over the extent of the superlattice. In summary, sample 8 appears to show very low levels of residual strain whereas the remaining superlattices show evidence of large strain fields.

The evolution of strain distributions during growth has been studied through in situ RHEED measurements performed on a single sample. Data were accumulated for a superlattice sample consisting of 200 repeats of $25 \AA \mathrm{CdTe}$ and $25 \AA \mathrm{ZnTe}$ grown on a CdTe buffer layer. Results suggest that in-plane lattice parameters were changing dramatically during the first one-half micron of growth (about 100 superlattice periods). After this point, however, the remaining superlattice periods appear to have grown according to a 
single in-plane lattice constant intermediate to those of $\mathrm{CdTe}$ and $\mathrm{ZnTe}{ }^{1+}$ This result is consistent with the apparent dramatic reduction of defect densities away from the buffer layer interface, observed through photoluminescence and TEM. It is also consistent with the high average levels of strain observed through $\mathrm{x}$-ray diffraction

\section{DISCUSSION}

Our data provide a fairly sensitive test of critical thickness theory for large lattice mismatch. Figure 5 shows plots of several theoretical models of the critical thickness at which a thin epitaxial layer will break away from a lattice-mismatched substrate. Each model uses an input value of Poisson's ratio and parameters describing the inclinations, densities, and Burger's vectors of the dislocations under consideration. CdTe parameters were used in our calculations. Substituting $\mathrm{ZnTe}$ parameters yields critical thicknesses within $7 \%$ of these values. The earliest model shown, due to Van der Merwe, ${ }^{15}$ relies on an energy balancing argument. According to this argument, strain is accommodated elastically until it becomes energetically favorable to form a network of dislocations sufficient to relieve mismatch stresses. This model is readily adapted to the types and densities of dislocations typically seen in specific systems, but dramatically underestimates critical thicknesses for most systems of small mismatch. ${ }^{16,17}$ A later model, due to Matthews and Blakeslee, ${ }^{18}$ matches forces which can be accommodated by threading dislocations to stresses due to lattice mismatch. Equating these forces identifies a point at which strain

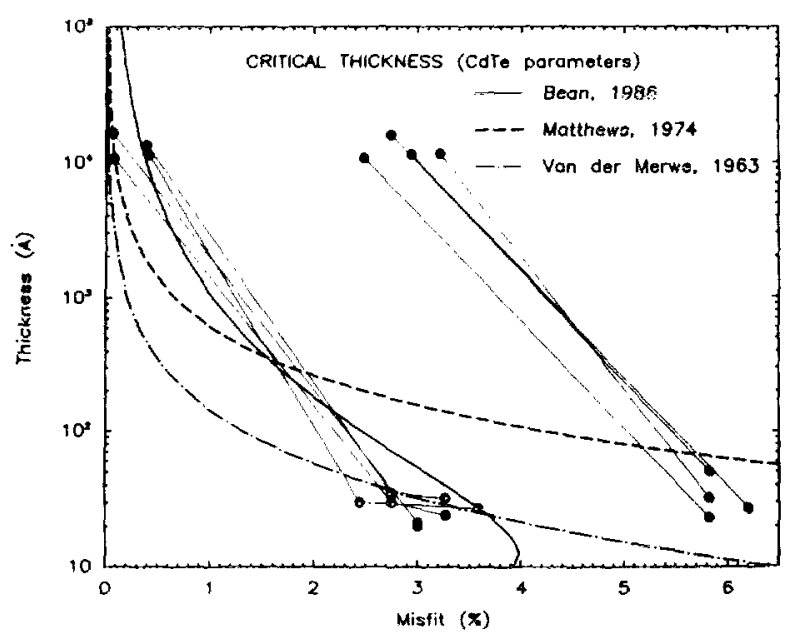

Fig. 5. Models due to Van der Merwe, Matthews, and Blakeslee, and People and Bean describing theoretical limits to misfit-free growth of a thin epitaxial layer as a function of lattice mismatch to substrate. Points corresponding to our sample thicknesses and misfits are also plotted. Each sample contributed several points, connected by lines, appropriate to the thicknesses and misfits of the individual layers in the superlattice and of the alloy of identical composition and thickness to the superlattice. Sample 2, for example, gives a point at $(2.47 \%, 1.08 \mu \mathrm{m})$ since this is a $1.08 \mu \mathrm{m}$-thick superlattice with a composition equivalent to a $\mathrm{Cd}_{0.57} \mathrm{Zn}_{0.43} \mathrm{Te}$ alloy, grown on a CdTe buffer layer $(2.47 \%$ mismatch). This point is connected to one at $(5.8 \%, 23 \AA)$ appropriate to the $23 \AA \mathrm{ZnTe}$ layers grown on the CdTe buffer. The poin derived from the individual CdTe layers, $(0 \%, 31 \AA)$, has not been plotted since the zero mismatch places no constraints on CdTe layer thickness Theory clearly predicts that this particular superlattice should exceed the critical thickness. This agrees with experiment.

J. Vac. Sci. Technol. B, Vol. 5, No. 4, Jul/Aug 1987 should be relieved by misfit defects. This model has met with mixed success. ${ }^{19}$ Bean and People ${ }^{19,20}$ have used energy balancing arguments to derive a critical thickness model which is highly implausible on a microscopic scale. The resulting curve is, however, in excellent agreement with data obtained for the Ge/Si system.

It has been suggested ${ }^{20-22}$ that superlattices should obey two critical thickness criteria. The degree to which a superlattice remains free of defects should depend in part on the thicknesses and misfit of each superlattice layer. In addition, it has been suggested that to be free of defects the entire superlattice should meet the critical thickness criteria appropriate to an alloy of the same thickness and volume-averaged composition. Accordingly, in Fig. 5 we have plotted sample thicknesses versus mismatch for each of the superlattices studied. Each sample contributed either two or three points, connected by lines for purposes of identification, appropriate to the thicknesses and misfits of the individual layers and the corresponding alloy. Sample 8 , for example, gives a point at $(0.07 \%, 1.64 \mu \mathrm{m})$, since this superlattice is $1.64 \mu \mathrm{m}$ thick with a $51 \% \mathrm{CdTe}, 49 \% \mathrm{ZnTe}$ volume-averaged composition, grown on a $\mathrm{Cd}_{0.50} \mathrm{Zn}_{0.50} \mathrm{Te}$ buffer layer $\left(a_{\mathrm{Cd}_{0.51} \mathrm{Zn}_{0.49} \mathrm{Te}} /\right.$ $a_{\mathrm{Cd}_{0.50} \mathrm{Zn}_{0.50} \mathrm{Te}}=0.07 \%$ misfit). This point is connected to others at $(3.0 \%, 21 \AA)$ and $(3.0 \%, 20 \AA)$ appropriate to the thickness and misfits of the individual CdTe and $\mathrm{ZnTe}$ layers, respectively. Four of the samples, grown on pure CdTe or $\mathrm{ZnTe}$ buffer layers, appear to exceed all critical thickness predictions. Those samples grown on alloy buffer layers lie in a more uncertain regime, however, with samples 5 and 8 meeting all but the constraints placed by the Van der Merwe model.

All of our samples have been observed to exceed the critical thickness. Sample 8 , which should have been closest to satisfying the critical thickness criteria, appears to show the smallest degree of residual strain. Each sample was handled similarly after growth, but sample 8 was grown several months prior to the others. This suggests the possibility that growth conditions played a role in distributing strain in these samples. Annealing experiments performed on $\mathrm{Ge} / \mathrm{Si}$ superlattice have demonstrated the metastable nature of these structures. ${ }^{23}$ In particular, annealing at temperatures higher than typically used during MBE growth results in the formation of substantial numbers of defects in these structures. It should not be surprising that such a system might be sensitive to even small changes in growth conditions. For this reason, we feel that a complete description of critical thickness cannot ignore growth parameters. While Van der Merwe's model presents a plausible lowest energy state for the system, the degree to which a sample displays a critical thickness in agreement with this prediction depends on its ability to acquire the activation energy necessary to reach this state.

\section{CONCLUSIONS}

The structure of a number of CdTe-ZnTe superlattices has been examined. Each of the samples studied has been grown beyond the critical thickness for generation of misfit defects, in disagreement with a number of theoretical models. Our data suggest that growth conditions play a role in 
determining the onset of defect formation. These conditions are neglected in present critical thickness models. We have evidence that defect densities drop dramatically within a micron of the superlattice/buffer-layer interface, regardless of the buffer layer used. The intense luminescence that results from this high-quality growth continues to make these structures exciting prospects for applications in optoelectronics.

\section{ACKNOWLEDGMENTS}

One of us (RHM) is grateful to IBM for financial support. We have benefitted from several valuable discussions with M. B. Johnson and P. M. Petroff. We also wish to acknowledge the technical assistance of S. Anlage and C.-R. Wie. Work at the University of Illinois was supported by the Defense Advanced Research Projects Agency under Contract No. MDA 903-83-K-025. The authors wish to acknowledge the partial support of DARPA/ONR under Contract No. N00014-86-K-0841.

'H. Fujiyasu, H. Takahashi, H. Shimizu, and A. Sasaki, in Proceedings of the 17th International Conference on the Physics of Semiconductors (Springer, New York, 1985), p. 539.

${ }^{2}$ M. Kobayashi, N. Mino, H. Katagiri, R. Kimura, M. Konagai, and K. Takahashi, Appl. Phys. Lett. 48, 296 (1986).

'R. H. Miles, G. Y. Wu, M. B. Johnson, T. C. McGill, J. P. Faurie, and S. Sivananthan, Appl. Phys. Lett. 48, 1383 (1986).
${ }^{4}$ G. Monfroy, S. Sivananthan, X. Chu, J. P. Faurie, R. D. Knox, and J. L. Staudenmann, Appl. Phys. Lett. 49, 152 (1986).

${ }^{5}$ M. D. Camras, J. M. Brown, N. Holonyak, Jr., M. D. Nixon, R. W Kaliski, M. J. Ludowise, W. T. Dietze, and C. R. Lewis, J. Appl. Phys. 54 6183 (1983).

${ }^{6}$ H. Jung, A. Fischer, and K. Ploog, Appl. Phys. A 33, 97 (1984).

'P. M. Petroff, C. Weisbuch, R. Dingle, A. C. Gossard, and W. Wiegmann, J. Vac. Sci. Technol. 19, 571 (1981).

${ }^{\star}$ R. N. Bicknell, N. C. Giles-Taylor, D. K. Blanks, R. W. Yanka, E. L. Buckland, and J. F. Schetzina, J. Vac. Sci. Technol. B 3, 709 (1985).

${ }^{9}$ Y. Hefetz, D. Lee, A. V. Nurmikko, S. Sivananthan, X. Chu, and J. P. Faurie, Phys. Rev. B 34, 4423 (1986).

${ }^{10} \mathrm{G}$. L. Bir and G. E. Pikus, Symmetry and Strain-Induced Effects in Semiconductors (Keter, Jerusalem, 1974).

${ }^{11}$ P. M. Petroff (unpublished).

${ }^{12}$ H. P. Klug and L. E. Alexander, X-Ray Diffraction Procedures For Polycrystalline and Amorphous Materials (Wiley, New York, 1974).

${ }^{13}$ International Tables for $X$-ray Crystallography (Kynoch, Birmingham, 1963 )

${ }^{14}$ G. Monfroy, X. Chu, M. Lange, and J. P. Faurie (to be published)

15. H. Van der Merwe, J. Appl. Phys. 34, 123 (1963)

${ }^{\prime \prime}$ C. A. B. Ball and J. H. Van der Merwe, in Dislocations in Solids, edited by

F. R. N. Nabarro (North-Holland, Amsterdam, 1983), Vol. 6.

17]. W. Matthews, in Epitaxial Growth, edited by J. W. Matthews (Academic, New York, 1968), Part B.

${ }^{18}$ J. W. Matthews and A. E. Blakeslee, J. Cryst. Growth 27, 118 (1974); 29 273 (1975); 32, 265 (1976).

${ }^{19}$ R. People and J. C. Bean, Appl. Phys. Lett. 47, 322 (1985).

${ }^{20} \mathrm{~J}$. C. Bean, in Silicon Molecular Beam Epitaxy, edited by E. Kasper and J. C. Bean (Chemical Rubber, Boca Raton, FL, 1987)

${ }^{21}$ M. Y. Yen, A. Madhukar, B. F. Lewis, R. Fernandez, L. Eng, and F. J. Grunthaner, Surf. Sci. 174, 606 (1986).

${ }^{22}$ T. C. Lee, M. Y. Yen, P. Chen, and A. Madhukar, Surf. Sci. 174, 55 (1986).

${ }^{23}$ A. T. Fiory, J. C. Bean, R. Hull, and S. Nakahara, Phys. Rev. B 31, 4063 (1985) 